\title{
EL TRIUNFO DE LAS CLASES MEDIAS. Dialéctica entre cambio social y urbanismo en Poblenou, Barcelona
}

\author{
José A. MANSILLA LÓPEZ \\ Observatori d'Antropologia del Conflicte Urbà (OACU) \\ joseamansilla@hotmail.com
}

THE TRIUMPH OF THE MIDDLE CLASSES. Dialectic between social change and urbanization in Poblenou, Barcelona

Resumen: El debate en torno a la gentrificación comenzó con la acuñación del término hace ahora 50 años. Desde entonces han surgido distintas corrientes teóricas que abordan la cuestión desde diferentes perspectivas. Algunas de las aproximaciones clásicas abundan en análisis rígidos que ocultarían las dinámicas generales de apropiación capitalista que se producen bajo el neoliberalismo. De este modo, la gentrificación se mostraría como una forma más de extracción de rentas monopolísticas del suelo urbano. Por otro lado, los análisis de las dinámicas de gentrificación olvidan, a menudo, la importancia de la cuestión de clase. El caso del Poblenou no es ajeno a ello. Objeto de intervención urbanística desde hace años, el barrio vive, hoy en día, una profunda transformación de su tejido económico y social. Un nuevo barrio, o nuevos barrios, que se crean y cambian en función de esta relación dialéctica.

Abstract: The debate about gentrification started after the coining of such a concept almost 50 years ago. From then on, several theoretical frameworks have been studying the issue from different points of view. Some of classical approaches could fall into rigid analysis that would hide the general dynamics of capitalist appropriation produced under neoliberalism. Hence, gentrification would be another way of extracting monopolistic rents from urban land. On the other hand, most of the researches about gentrification frequently forget the relevance of the class question. The case of Poblenou is no stranger to it. Object of urban intervention since years ago, the neighborhood lives nowadays a deep transformation of its social and economic fabric. A new neighborhood, or new neighborhoods, are being created and changed through this dialectic relationship.

Palabras clave: Transformación; Barcelona; Plusvalías; Gentrificación; Turismo Transformation; Barcelona; Surplus value; Gentrification; Tourism 


\section{La Ciudad Cambiante}

En septiembre de 2013, cuando casi llevaba un año realizando el trabajo de campo de mi tesis doctoral, ${ }^{1}$ tuve la suerte de poder mantener una conversación informal con una vecina, Eulalia ${ }^{2}$, sobre los cambios producidos en el Poblenou, Barcelona, durante las últimas décadas. Eulalia, señera activista y testigo de primera mano de la historia del barrio, me señalaba, cómo "esto en el año 1975-76 era un polvorín: MACOSA, Can Torras y la Flor de Maig? ${ }^{3}$ Yo iba a las manifestaciones con mi hija de un año y mi madre me decía 'ten cuidado', pero yo la llevaba muy fuerte agarrada del cuello"4.

Sin embargo, cualquiera que se acerque hoy en día al Poblenou puede comprobar que ya no es posible encontrar ni las fábricas ni los conflictos asociados que me recordara Eulalia tropezando, en su lugar, con diversos equipamientos públicos y privados, abundantes promociones inmobiliarias y una economía centrada en el turismo y los servicios. La transformación del barrio, antiguo bastión industrial y obrero de la Barcelona fabril, ha sido notoria.

Las ciudades son siempre espacios en constante mutación, por lo que no hay que caer en cierto tipo de discurso nostálgico, o incluso conservador, sobre un supuesto pasado popular de calles y barrios. Ahora bien, eso no quiere decir que éstas no sean el escenario donde operan determinadas dinámicas de poder, como los procesos de gentrificación, que influyen de manera poderosa en su transformación física y social. En el presente texto me centraré en las transformaciones producidas en el Poblenou comenzando con una provocación: el barrio del Poblenou nunca ha sufrido un proceso de gentrificación, en el sentido "clásico" del término.

\section{¿Un Poblenou Gentrificado?}

Desde su creación por la socióloga inglesa Ruth Glass (1964), hace ahora 50 años, la gentrificación ha logrado una relevancia creciente, tanto que hace ya tiempo que escapó de los círculos universitarios para pertenecer al lenguaje común. Ruth Glass definía la gentrificación, refiriéndose al Londres de la época, como aquellos cambios ocurridos en determinados barrios, invadidos por las clases medias y altas, que habrían derivado en un desplazamiento de las clases populares que hasta entonces lo habitaban, cambiando totalmente el carácter del mismo (Ibídem). Gentrificación es un anglicismo que proviene del sustantivo gentry, cuya traslación al castellano sería la de alta burguesía o clase alta, y que vendría a ser aquella nueva aristocracia terrateniente a caballo entre la vieja nobleza feudal y la incipiente burguesía industrial. Así, la gentrificación supondría un proceso (el sufijo -ción en castellano implica siempre acción) en el que el resultado final sería el aburguesamiento -algunos autores prefieren llamarlo elitización (García Herrera, 2001)- de un área determinada debido a cambios ocurridos en el mercado de la vivienda.

El patrón clásico (Treanor, 2004) de una dinámica/acción de gentrificación sería el si-

\footnotetext{
1 Durante mi trabajo de campo utilicé distintas técnicas etnográficas (entrevistas, diálogos informales, participación en acciones directas, etc.) para mi aproximación al objeto de estudio, esto es, los movimientos sociales y la memoria colectiva del barrio del Poblenou. La duración total del mismo fue de 19 meses, desde noviembre de 2012 a junio de 2014.

2 Para mantener el anonimato de las personas entrevistadas, todos los nombres han sido cambiados.

3 Material y Construcciones, S.A. (MACOSA) y Can Torras fueron complejos industriales que existieron y pervivieron en el barrio hasta los años 80-90 del pasado siglo. La Flor de Maig es una antigua cooperativa de consumo de la cual se darán más detalles en próximos párrafos.

4 Conversación Informal Eulalia (CIE), Poblenou, 23/09/2013.

5 Por otro lado, es necesario aclarar, ya desde un principio, que el hecho de que este sea el relato más popular no significa que sea el único. De hecho, los procesos de desvalorización/revalorización de un área determinado pueden darse de diversas maneras, tal y como se han encargado de mostrar, entre otros Fernández (2012). Por
} 
guiente: un barrio céntrico de una ciudad, casi siempre estadounidense o europea, sufre un proceso inicial de abandono por los grupos sociales acomodados que solían vivir allí. Las necesidades de relocalización industrial del capital y la mano de obra, la terciarización del tejido económico (Harvey, 1989. Lefebvre, 1972), la popularización del uso del automóvil y las mejoras en el transporte público, unido a ciertas ideas vinculadas a la necesaria higienización del espacio urbano y la búsqueda de áreas abiertas (Sennet, 1997), llevan a estos grupos a buscar su residencia en las periferias. Estas áreas abandonadas se ven en un principio copadas por grupos de clase trabajadora, elementos del lumpen, familias monoparentales, minorías étnicas (Rose, 1984), etc., con poco poder adquisitivo, en busca de vivienda barata, lo que contribuye, aun más, a acelerar su proceso de degradación. Este mismo objetivo es el que buscan determinados colectivos, como artistas y artesanos, que mantienen un tipo de vida alejado de las prescripciones de las sociedades capitalistas contemporáneas, por lo que necesitan espacios donde poder desarrollar sus actividades a precios bajos. Posteriormente, debido a circunstancias vinculadas, por ejemplo, a cambios en los gustos y pautas de consumo social, las nuevas clases medias y altas se sienten atraídas por esos ambientes de carácter bohemio, lo que provoca una presión al alza en el mercado del suelo, expulsado a la vanguardia inicial, junto a los restos de grupos populares, y gentrificando el barrio. Ejemplos clásicos serían los expuestos por Richard Lloyd y sus neobohemians (2002) de Wicker Park, en Chicago, o los descritos por Jon Caulfield en su "Gentrification and desire" (1989) para los barrios de Toronto. Es evidente que este proceso, pese a sus más que evidentes similitudes en el resultado final, no es el que se ha producido en el Poblenou, un territorio que ha vivido profundos cambios en su entramado urbano, aunque básicamente debidos a las fuertes intervenciones realizadas en el barrio por parte de los diferentes niveles de la administración pública del Estado español (Capel, 2005 y 2007. Delgado, 2005 y 2007. Marrero, 2003. Telló, 1993), pero que no responde a este relato.

Por todo lo anterior, mi primera aproximación a las transformaciones ocurridas a lo largo de las últimas décadas en el barrio la realizaré acudiendo a conceptos económicos clásicos (algo de lo que no queda excluida la gentrificación, como veremos más adelante). Me refiero a aquello que Marx denominaba, cuando hablaba de los distintos tipos de renta, como "renta monopolista" y que David Harvey recoge en su conocida obra Urbanismo y Desigualdad Social (1977) en referencia al mercado de suelo urbano. Este tipo de renta es aquella que surge cuando es posible cobrar un precio monopolista por un determinado bien o servicio y se determina, de forma exclusiva, por la relación entre el deseo de compra y la capacidad de pago de los compradores, independientemente del costo de producción del producto o su valor. A un nivel más amplio, asistiríamos aquí a un enfrentamiento entre valor de uso y valor de cambio. Podemos ver la diferencia entre ambos tipos de valor mediante el ejemplo de la vivienda. Así, el valor de uso de una vivienda se manifiesta en la necesidad de las personas de acceder a un lugar donde poder vivir y desarrollar una vida familiar e íntima, mientras que, por otro lado, el valor de cambio aparecería cuando dichas viviendas son vendidas en el mercado inmobiliario a precios establecidos por este. Las diferencias y la relación entre uno y otro, originan una tensión, una contradicción, así como la posibilidad de obtener ciertas rentas monopólicas por parte de los propietarios del suelo donde dichas viviendas se emplazarían. En un caso extremo, como hemos visto en la reciente crisis económica, se podría llegar a generar una burbuja inmobiliaria que conduciría al sistema a la necesidad de reestructurarse (Harvey, 2014).

De esta forma, la posibilidad de imponer un precio monopolista crea, para el propietario

otro lado, las definiciones en torno al concepto han supuesto encendidos debates, Arbona (2014). En el presente texto se adopta esta descripción inicial por motivos de la propia estructura y desarrollo del mismo, tal y como podrá verse a continuación.

6 El Capital, 2003. 
del suelo, la oportunidad de obtener una renta monopolista ${ }^{7}$.

\section{A la Búsqueda de la renta Monopolística: Tres Ejemplos}

El actual Distrito de Sant Martí, en el que se encuentra el barrio del Poblenou, ha sido objeto de la búsqueda de este tipo de rentas $^{8}$ desde tiempos inmemoriales. Josep María Huertas Claveria, Jaume Fabre y José Martí, en su imprescindible Tots els barris de Barcelona (1976), relatan cómo justo después de la Guerra de Sucesión, hace ahora 300 años, ya se produjeron algunos ejemplos de movimientos especulativos en el entorno de la, en aquel tiempo, parroquia de Sant Martí de Provençals. Por aquel entonces, gran parte del barrio eran terrenos públicos destinados a pastos y ganadería. Con ellos se trataba de garantizar el abastecimiento de trigo y leche y derivados para la población de la ciudad de Barcelona. Eran amplias zonas de terreno pantanoso (de las que todavía quedan referencias en el nomenclátor del barrio, como las calles Llacuna o Joncar) que pasaron, tras la Guerra, a depender, tanto en administración como en propiedad, del nuevo soberano, que lo gestionaba a través de un Intendente. Durante más de 100 años, los diferentes ocupantes de este cargo fueron liquidando unos terrenos que, por su localización y características (abundancia de agua y arenas), eran de alto interés para la naciente industria de indianas. Estos terrenos se vendieron inicialmente a precios bajos, de forma que los empresarios aprovecharon para hacer suculentos negocios con el suelo urbano intramuros que habían ocupado hasta entonces. Independientemente de la titularidad de los terrenos (aunque es imposible negar su importancia) el precio que llegó a alcanzar el suelo del actual barrio fue un ejemplo claro de renta monopolística, pues las características del mismo (no olvidemos que posteriormente se encontraría en una localización inmejorable con respecto al transporte ferroviario y al puerto) permitían imponer una renta de estas características a la incipiente necesidad de suelo industrial. Precisamente comienza aquí la transformación del tejido social del asentamiento, ya que, junto con las industrias, llegaron los obreros y obreras que compartirían el espacio junto a agricultores, ganaderos y pescadores.

Dando un enorme salto en el tiempo, llegamos a otro de los ejemplos de puesta en marcha de mecanismos de extracción de rentas monopolísticas del suelo del Poblenou. En el año 1966 el barrio vive un cierto declive industrial. Lo que había sido conocido como el Manchester catatán fue fracturando y trasladando a las afueras su tejido industrial, muchas veces obsoleto. Es entonces cuando aparece, con la complacencia del Alcalde franquista de entonces, Josep María de Porcioles, el Plan de la Ribera y con él el debate sobre la reordenación del barrio como territorio urbano. El Plan desarrollado por una sociedad de empresarios, Ribera, S.A., planeaba la construcción, dada la cercanía de la costa (he aquí la oportunidad de rentas), de amplios complejos de viviendas de gama alta. Además, entre sus objetivos estaba la recuperación de las playas y el litoral; la eliminación del trazado ferroviario existente junto a la línea costera y el impulso a la terciarización productiva mediante la estimulación de la aparición de pequeño comercio junto a grandes centros comerciales. El Plan afectaba a un total de 234 hectáreas comprendidas entre la Maquinista, al este en la Barceloneta, y Sant Adrià del Besòs, al oeste ${ }^{9}$. Se trató de una operación montada por em-

\footnotetext{
7 Este tipo de renta es, a veces, confundido con las rentas diferenciales o, incluso, con las rentas de localización, las cuales están determinadas por la distancia existente de un espacio isótropo determinado al mercado, pero el mismo Harvey las reserva para casos muy específicos (Harvey, 1977).

8 La definición de renta que seguiremos aquí será la dada según el propio David Harvey citando a Marx en El Capital y que dice que "la propiedad territorial presupone el monopolio de ciertas personas que les da derecho a disponer sobre determinadas porciones del planeta como esferas privativas de su voluntad privada, con exclusión de todos los demás. Partiendo de esto, se trata de explotar el valor económico, es decir, de valorizar este monopolio sobre la base de la producción capitalista" (1977: 187)

9 Las empresas que participaron en la elaboración del Plan fueron: Catalana de Gas (posterior Gas Natural),
} 
presarios que querían abandonar el barrio, aunque no su actividad, y a la vez conseguir una fuerte revalorización de sus terrenos mediante el desarrollo de lo que, entonces, se llamó la Copacabana barcelonesa ${ }^{10}$. El plan contó, en su momento, con una fuerte oposición vecinal y finalmente nunca se llevó a cabo, aunque quizás es posible afirmar que sirvió como laboratorio de pruebas de la colaboración público-privada que caracterizaría una futura forma específica de hacer urbanismo. Por otro lado, algunos elementos fueron posteriormente, ya acabado el franquismo, recuperados y ejecutados (Mansilla, 2014a).

El último de los ejemplos de extracción de rentas monopolísticas que presentaremos será el del Plan 22@. Este plan constituyó, en su momento, la transformación más importante de la ciudad ya que llegó a afectar a casi 116 hectáreas de suelo industrial, equivalente a casi 120 manzanas del Eixample. El nombre 22@, trasladado al territorio como Distrito 22@, provenía de la recalificación de los suelos calificados como industriales en el original Plan General Metropolitano de 1976, los cuales recibían la etiqueta catastral 22a. El Plan se enmarcó dentro del documento de Modificación del Plan General Metropolitano del año 2000, el cual se pretendía que fuera flexible ya que, como el mismo reconocía, la complejidad de las transformaciones, así como las operaciones que pretendía impulsar el Ayuntamiento de Barcelona, junto a aquellas desarrolladas por la iniciativa privada, así lo exigían. Tal y como recogía su introducción, la finalidad del Plan era enfrentarse al reto de la nueva economía proponiendo al Poblenou como "la principal plataforma económica y tecnológica de Barcelona, Cataluña y España, en la perspectiva del Siglo XXI" (Ajuntament de Barcelona, 2000). La idea era facilitar, mediante la normativa urbanística, una cierta recuperación industrial basada en las nuevas tecnologías y la economía del conocimiento. Sin embargo, en un trabajo reciente, Charnock et al (2014) demuestran que, tras el esfuerzo realizado por el Ayuntamiento de Barcelona de transformar la economía local, no estaba más que el diseño institucional de la posterior apropiación, por parte del capital inmobiliario, de las rentas producidas por el suelo de la ciudad, hecho que se produjo mediante la transformación de dicho suelo en activo financiero. De esta forma, muchos de los desarrollos inmobiliarios llevados a cabo en el 22@ se basaron en la venta de edificios que no tenían una garantía última de ser ocupados y que, además, tampoco contaban con una definición clara sobre qué tipo de actividad se entendía como relacionada con la economía del conocimiento. Esto llevó, según dichos autores, a que solo el 30\% del suelo ocupado en el 22@ en el año 2005 estuviera bajo el paraguas de las nuevas tecnologías, mientras que casi el $70 \%$ lo copaban hoteles y empresas vinculadas a los seguros, los servicios financieros y el marketing (Ibídem).

\section{El Triunfo de las Clases Medias}

Ahora bien, y volviendo al tema de la gentrificación, hasta ahora hemos señalado el carácter ajeno a este proceso de las transformaciones llevadas a cabo en el Poblenou basándonos en la popular y ampliamente aceptada definición dada por Ruth Glass para el Londres de inicios de los años sesenta, y en un relato muy rígido y tópico de funcionamiento del sistema. Sin embargo, tal y como señala Tom Slater (2011), esta perspectiva puede ser muy miope, entre otras cosas porque nos encontramos hoy en día ante unos fenómenos urbanos bastante diferentes, y mucho más complejos, a los observados hace 50 años. De este modo,

Motor Ibèrica, Maquinista Terrestre i Marítima, Foret, Crèdit i Docks, Hijo de E. F. Escofet, Hidroelèctrica de Catalunya, MACOSA, Martini Rossi, Jorge Wahl y RENFE, y contaban con el apoyo de varios bancos y cajas de ahorro (Huertas Claveria, Fabre y Martí, 1976. Alibés et al, 1975).

10 La expresión "Copacabana barcelonesa" se atribuye al Alcalde Porcioles. En este sentido, y rebatiendo las teorías que hablan de varias etapas en el urbanismo llevado a cabo en Barcelona, en el año 1999 aparece un artículo en el diario El País donde precisamente se hace referencia a la "alegría" de la ciudad al haber conseguido realizar una Copacabana Barcelonesa (Arroyo, 1999), precisamente 33 años después de haberse planteado la idea inicial y con una administración municipal "democrática". 
definir las dinámicas de transformación de un barrio basándonos únicamente en el estudio del mercado de la vivienda destinado a las clases medias, no solo no es correcto desde el punto de vista analítico, sino que puede ser políticamente muy conservador. Así, la gentrificación sería parte de un proceso mucho mayor que el de la revitalización, regeneración y rehabilitación residencial, ya que contempla importantes elementos relativos a la transformación y el conflicto entre clases sociales (ibídem).

Como señalara el geógrafo Neil Smith (2002), la incapacidad de acceder a una vivienda para las clases populares y la catástrofe personal y familiar que suponen el desplazamiento, el desalojo y los desahucios, son síntomas de ciertas disposiciones institucionales, entre las que encontramos la exaltación de la propiedad privada y el libre mercado, que favorecen la creación de ambientes urbanos al servicio de la acumulación del capital. Esta acumulación, por su parte, se produciría a expensas de necesidades sociales tales como la vivienda y la vida en comunidad, esto es, del valor de cambio sobre el valor de uso. ${ }^{11}$

De esta forma, ampliando la perspectiva y bajo una mirada dialéctica, los procesos de gentrificación podrían definirse, desde un punto de vista de cambio social, como aquellos en los que se produce una transformación de un vecindario de bajo estatus social en otro de clase media o media-alta, y desde un punto de vista económico, como un eslabón más en la extracción de rentas del suelo urbano. Y para que esto ocurra no es necesario que se produzca, de forma estricta, el tradicional desarrollo de la gentrificación ${ }^{12}$ basado en el abandono-degradación-atracción-revalorización-desplazamiento de zonas céntricas que encajaría perfectamente en el marco clásico del diferencial de renta, sino que también puede ocurrir mediante la construcción de viviendas nuevas (adosados, torres de apartamentos, etc.); de cambios en el sistema productivo (oficinas, tiendas, restaurantes, hoteles, etc.); en localizaciones no céntricas (puertos, antiguas zonas industriales, etc.); fuera de las ciudades (zonas rurales y costeras), etc. (Shaw, 2008). El elemento fundamental sería que, en el transcurso de la acción, la ciudad y sus barrios dejarían de ser un espacio habitable y pasarían a considerarse como espacios consumibles.

Así, los procesos de transformación del entramado urbano que ha vivido el barrio del Poblenou en los últimos años responderían, más bien, a lo que autores como Paul Treanor (2004) han denominado brownfield redevelopment. Este tipo de redesarrollo consistiría en la construcción, o actualización de antiguos edificios, en terrenos anteriormente destinados a usos industriales, infraestructuras de transporte, etc., con la finalidad de ser dedicados a usos no industriales, como viviendas, oficinas, tiendas, hoteles o centros de servicios diversos (ibídem). De esta forma, mediante los cambios introducidos en el entramado urbano se originarían importantes transformaciones en su tejido social. Para finalizar, recordar a David Harvey cuando señala que,

"The exchange value of housing is not, however, fixed. It fluctuates over time according to a variety of social conditions and forces. To begin with, it is not independent of the exchange values of surrounding houses [...] Improvements in the neighbourhood (for example gentrification) will increase the value of my house even thought I myself have invested nothing. The housing market is

\footnotetext{
11 Como sabemos, este mismo autor desarrolló, a finales de los años setenta del pasado siglo, una de las perspectivas más interesantes para adentrarnos en el análisis de los procesos de gentrificación. Se trataría de la Teoría del rent gap, o diferencial de renta, y que podríamos definir como aquella diferencia existente entre la renta capitalizada del suelo bajo su actual uso y el nivel de la renta potencial del mismo (Smith, 2012). Esta aproximación teórica ha recibido numerosas críticas, como la elaborada por David Ley basada en la demanda de espacios residenciales céntricos por las nuevas clases medias, u otras que atacarían su postura excesivamente economicista y le reclaman haber olvidado elementos de carácter cultural y social.

12 En este sentido comparto la perspectiva del antropólogo Marc Morell cuando define estas etapas como "el trabajo de la gentrificación" en sus diferentes fases. Ver Morell, 2013.
} 
characterised by what economist call 'externality' effects” (2014: 19).

Es decir, el valor de cambio del suelo urbano se encuentra determinado, principalmente, por cuestiones de carácter externo al mismo, por las características de su entorno y su localización en la estructura urbana, esto es, y tal y como hemos visto antes, por su capacidad de generar rentas monopolísticas. A estas cuestiones externas, efectos externos o externalidades, entre las que encontraríamos elementos físicos y tangibles, tales como aeropuertos o bocas de Metro, la cercanía del mar o la montaña, etc., habría que sumarle los intangibles, un capital simbólico producido, a veces, por aquellas clases sociales e individuos que serán las principales perjudicadas en la posterior dinámica gentrificadora (Fernández, 2012. Mansilla, 2014c). Ejemplo de ello lo tenemos en el barrio barcelonés del Raval, antiguo Chino, donde los "badulaques" de indios y paquistaníes, la prostitución callejera, los colmados tradicionales y sus parroquianos, o su ambiente nocturno y canalla, en definitiva, su capital simbólico, es transformado en plusvalías y estas finalmente captadas por terceros, generalmente intereses inmobiliarios, desplazando a la población local.

Por tanto, ¿podríamos ver la gentrificación como el resultado de una forma concreta de obtención de plusvalías, en las que juegan un papel fundamental dos de las cuestiones anteriormente resaltadas: las rentas monopolísticas y la cuestión de clase?

\section{¿Clases?, ¿qué clases?}

Para entrar de lleno en la caracterización de los cambios de clase concernientes al Poblenou es necesario hacer alguna aclaración inicial, sobre todo aquella relativa a la propia conceptualización de lo que entendemos por "clase" y su relación con las cuestiones urbanas y la gentrificación. Fijaré inicialmente la atención en la consideración llevada a cabo por David Harvey cuando nos señala que actualmente el concepto clásico de clase escapa del área productiva (fábricas y empresas) y lleva la lucha contra la explotación a la ciudad como ámbito de la producción y reproducción del capital (Harvey, 2001, 2013 y 2014). En este sentido encontramos a los científicos sociales del denominado Movimiento Operario, del post operaismo (del que son ejemplos significativos Negri y Hart), así como las consideraciones de Jean Paul Gaudemar (1982) sobre las ciudades como auténticas fábricas sociales donde los fenómenos netamente urbanos deben ser controlados, como controlados y planificados están todos los aspectos de una fábrica. En Barcelona, el geógrafo Pere López Sánchez (1990) abunda en esta línea señalando como, en las sociedades capitalistas contemporáneas, al vaciado de las industrias le ha seguido la ampliación del complejo fábrica y su desplazamiento a un plano superior de carácter espacial, la ciudad. De hecho, y tal como señala el mismo Harvey, "The higher wages workers may get through class struggle in the workplace can all too easily be snatched back by the landlord, the credit card companies, the merchants, to say nothing to the taxman" (2014: 54) ${ }^{13}$. En este sentido, y volviendo al trabajo de Marc Morell, y a su concepto de urban labor o trabajo urbano, en relación con la gentrificación, los distintos grupos sociales que intervienen en las distintas fases del trabajo de la gentrificación (desvalorización, valorización, etc.) conformarían una misma clase ${ }^{14}$

13 La extracción del excedente, o plusvalía, que pudiera ser compartida con los trabajadores a consecuencia de su lugar en la cadena productiva (mediante el salario), se completa con el llevado a cabo en su lugar de reproducción mediante el pago del alquiler, las comisiones bancarias, servicios básicos como el agua, etc. Estaríamos, por tanto, ante una de las contradicciones que muestra el capitalismo, altamente patente desde el cambio de política económica (desde el keynesianismo de postguerra a las medidas neoliberales de los años setenta) ante la necesidad de seguir circulando y acumulándose y que nos enseña, además, la necesidad de repensar la unidad y el conflicto entre clases, tradicionalmente articulado en torno al lugar de trabajo. Harvey, 2014.

14 En este sentido es interesante resaltar como es frecuente, en la literatura científica y algunos medios de comunicación norteamericanos, encontrar referencias a la ausencia de conflictos entre "gentrificadores" y "gentrifica- 
que se encontraría, precisamente, trabajando en la cadena de producción de valor (Morell, 2013). Así, los que se apropiarían de estas plusvalías producidas por los trabajadores urbanos, y siempre con la connivencia, cuando no el papel activo del Estado, sería el capital inmobiliario y los especuladores que explotan las rentas de monopolio, esto es, los diferenciales de renta generados (Franquesa, 2007).

Pero el hecho de que diferentes grupos sociales, a través del trabajo urbano, participen conjuntamente en la cadena de producción de valor que supone todo proceso de gentrificación, no significa que no se produzcan grandes desigualdades en un mismo barrio o ciudad, así como segregación social y espacial, esto es, desplazamientos de clase, dependiendo, en esta ocasión, de su posición en el sistema productivo (trabajadores altamente vs escasamente remunerados, por ejemplo). Es más, dicha consideración, pese a ver válida y compartida, no se presenta como muy útil a la hora de analizar la dialéctica entre el cambio social y el urbanismo que se ha producido en el Poblenou. La necesidad de abordar ciertos aspectos, como la vinculación de los procesos de gentrificación con la mercantilización de los modos de vida, las pautas de consumo, etc., en definitiva, con la aparición y triunfo de las clases medias y medias-altas, hace necesaria la búsqueda de una definición más acorde a esta investigación.

La sociología nos propone que las clases sociales pueden llegar a mesurarse y definirse basándonos en una serie de criterios objetivos vinculados a, por ejemplo, los niveles de ingreso, tipo de vivienda y de trabajo, estilo de vida, conducta electoral, nivel educativo, etc. (Giner, 1981). Se trataría, no tanto de términos propiamente analíticos, unidades estructuralmente o funcionalmente definidas, sino, más bien, de una aproximación escalar y descriptiva, útil para el estudio y análisis de sociedades dinámicas (Leeds, 1994).

De esta forma, para el análisis de los cambios de clase acontecidos en el Poblenou, realizaré inicialmente un análisis cuantitativo basado en cuatro variables estadísticas: población, nivel de ingresos (renta familiar), vivienda (precio del $\mathrm{m}^{2}$ ) y nivel de formación (estudios alcanzados). Entenderé que aquellos grupos sociales que alcancen mayores posiciones en los indicadores reseñados se corresponderán con aquellas clases medias y medias-altas que se busca definir. Posteriormente enfrentaré los resultados obtenidos con información de carácter cualitativo, a partir de los datos obtenidos durante el trabajo de campo etnográfico.

\section{El Poblenou Empírico}

La ciudad de Barcelona se encuentra dividida en 10 Distritos, los cuales, desde el año 2007, a su vez se hallan fraccionados en 73 barrios. Fue a partir de ese momento que el Poblenou, considerado históricamente un único barrio, fue subdividido en 5 unidades diferentes: El Parc i la Llacuna del Poblenou, la Vila Olímpica del Poblenou, el Poblenou, Diagonal Mar i el Front Maritim del Poblenou y Provençáls del Poblenou ${ }^{15}$. Estos nuevos barrios, conjuntamente con el Besòs i el Maresme, el Camp de l'Arpa del Clot, el Clot, Sant Martí de Provençáls y la Verneda i la Pau constituyen el Distrito 10 de Sant Martí16.

La subdivisión llevada a cabo en el territorio histórico del Poblenou obedece, según el Ayuntamiento de la ciudad, a la necesidad de establecer una delimitación en barrios significativos desde el punto de vista urbanístico y social, y como marco para el desarrollo de actuaciones urbanas y dotación de determinados servicios y equipamientos (Ayuntamiento de Barcelona, 2006). Sin embargo, como veremos más adelante, esto puede haber conducido a la creación de distintas unidades socio-espaciales que han roto con la tendencia política previamente establecida desde las instancias municipales de soslayar la creación de "guetos"

dos", optando por mostrar una visión optimista donde todos ganan. Davidson, 2014 y Nevius, 2014.

15 La frontera del Poblenou, antes del año 2007, coincidía aproximadamente con los límites establecidos actualmente por el Parc de la Ciutadetalla al Sur, la Avenida Diagonal al oeste, y la calle Josep Pla al norte.

16 http://www.ben.cat/estadistica/castella/terri/index.htm 


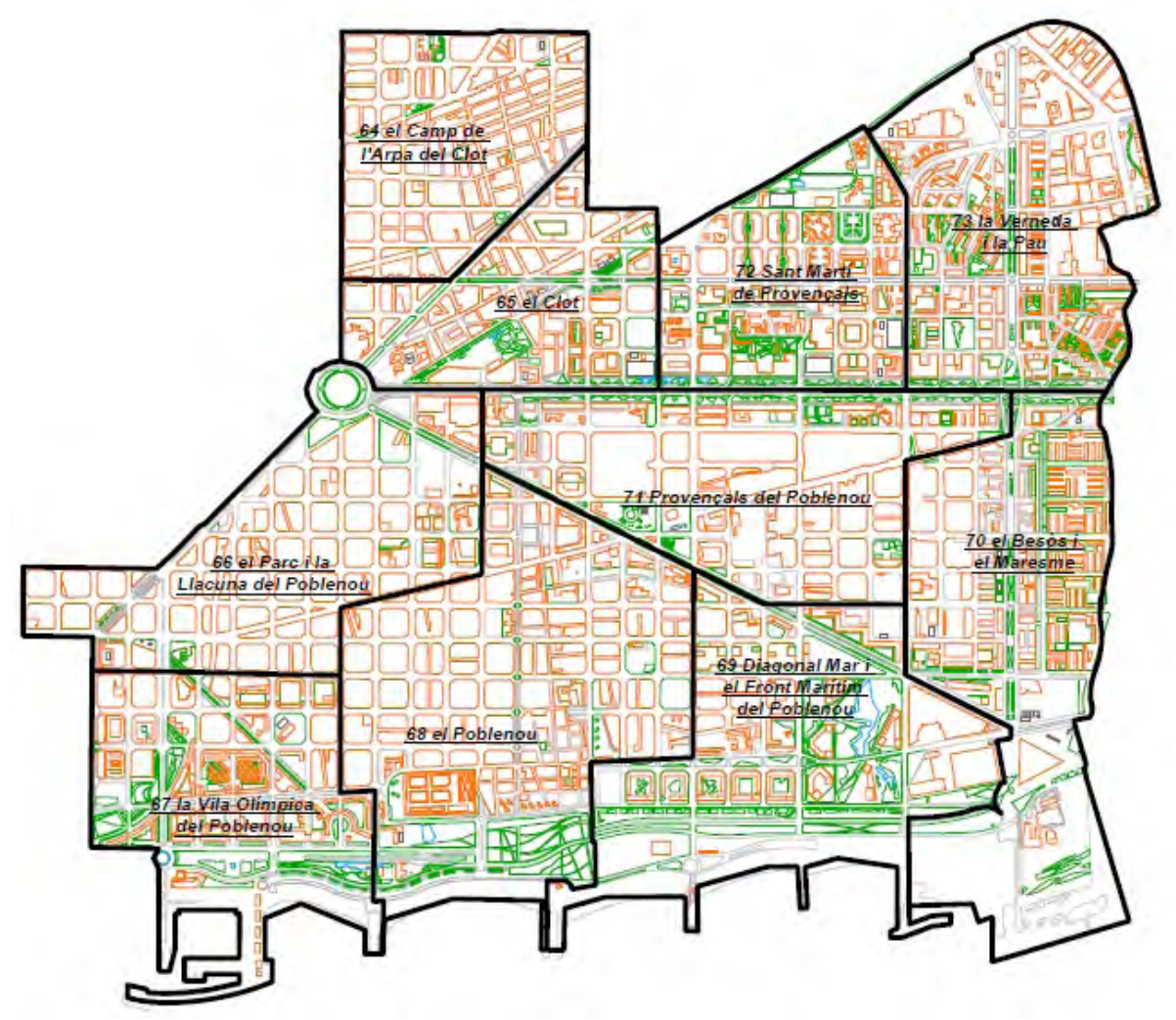

\section{Mapa n¹. Los barrios del Distrito de Sant Martí Fuente: Ajuntament de Barcelona}

socialmente homogéneos, así como dotar de continuidad a las nuevas áreas con las antiguas evitando la especialización funcional (Garcia-Ramón y Albet, 2000).

Comenzaré por analizar la población del Distrito de Sant Martí17 ${ }^{17}$ la cual ha venido creciendo de forma constante durante el periodo comprendido entre el año 1991 y el 2012. En estos 21 años, la población total se ha visto incrementada en un total de 19.211 habitantes, lo que supone un aumento de casi el $9 \%$. Durante los 10 primeros años de la serie se produjo una importante pérdida de población en el área, de 6.272 personas, tendencia que se ve posteriormente modificada a partir del año $2001^{18}$. La distribución por sexo de la población se ha mantenido constante, siendo a finales de 2012 , de un $51,7 \%$ de mujeres frente a un $48,3 \%$ de hombres.

17 La fuente principal de información es el Departament d'Estadistica de l'Ajuntament de Barcelona. La información disponible para variaciones interanuales amplias (1991-2012) sólo está referida a la totalidad del Distrito de Sant Martí. Los datos relativos al Poblenou, uno de los 10 barrios en los que se divide dicho Distrito, contemplan únicamente la serie 2010-2012. Por esto, en determinadas ocasiones, será necesario hacer extrapolaciones de la situación del Distrito a la del barrio.

18 En este año ya eran una realidad la promoción de viviendas del Front Maritim, de las mayores que se han construido en el Distrito, y se estaban construyendo las de Diagonal Mar con las que, posteriormente, constituiría su propio barrio. Estas promociones se llevaron a cabo en antiguos terrenos industriales (brownfield redevelopment), concretamente en los de Catalana de Gas, Renfe y MACOSA. De hecho, según una noticia aparecida en el diario El País, para ese mismo año, solo las viviendas en proyección ya supondrían una afluencia total de 44.645 vecinos. Ver Utrera, 2001. 
Descendiendo más al detalle, por barrios, la fotografía actual del Distrito muestra que los barrios más poblados son los de, por este orden, el Camp del Arpa del Clot, el Poblenou, la Verneda i la Pau y el Clot, con 38.232, 32.816, 29.201 y 27.201 habitantes respectivamente. Los menos poblados son la Vila Olímpica del Poblenou con 9.322 y Diagonal Mar i el Front Maritim con 12.701 habitantes. El Poblenou, por su parte, ha visto incrementar su población en los últimos tres años considerados (2010-2012) en un 4,6\%.

Es posible observar que los incrementos de la población han sido constantes pero, ¿cómo se distribuye este incremento poblacional según grupos de edad? A nivel Distrito, para la serie anual 1991-2012, son llamativos los cambios producidos en el grupo de jóvenes entre 15-24 años, los cuales pasan de representar el 15,7\% del total de la población en 1991, a solo un $8,7 \%$ en 2012 . La pérdida de importancia de ese grupo de edad es progresiva. El resto de los grupos sufren cambios, aunque en menor cuantía, destacando un cierto envejecimiento de la población que se muestra, incluso, en un incremento de los porcentajes relativos al grupo de edad de mayores de 65 años, que pasa de representar el 15,4\% al 19,6\% y en el incremento del grupo de adultos, entre 25-64 años, que sube un 5,2\%.

Más en detalle, en base a edades quinquenales, los grupos de edad comprendidos entre los 15 y 29 años $(15-19,20-24,25-29)$ sufren una caída del 23,4\% al 10,9\% para el inicio y el final de la serie respectivamente (1991-2012). Esto podría suponer un dato relevante a la hora de evaluar la existencia de un supuesto desplazamiento de este sector de la población, y mostraría que los jóvenes del barrio, ya sea por el precio alcanzado por los pisos y casas en el barrio, por la dificultad a la hora de encontrar un trabajo, o al salario medio que reciben en sus respectivos empleos, no podrían acceder a una vivienda en el mismo.

Para confirmar este extremo, nos referiremos a algunos datos aportados por el trabajo etnográfico. Así, en una entrevista llevada a cabo al responsable de uno de los Casals de Barri $^{19}$ del Poblenou, preguntado por cómo evaluaba él los cambios producidos en el barrio en los últimos años, obteníamos la siguiente respuesta:

"Mucha gente se ha tenido que marchar. Aunque no es un fenómeno único, ha pasado en otros barrios de Barcelona. En la transformación que ha sufrido el Poblenou hay muchas cosas positivas, pero no se puede ver todo así. En el camino se ha quedado mucha gente. Por ejemplo, los jóvenes. Hay muchos jóvenes que se han tenido que marchar porque, aunque ahora están bajando, algo, no mucho, los precios de la viviendas, los niveles alcanzados expulsaron a mucha gente y sobre todo a los jóvenes. Conozco muchos casos. Todo el proceso de especulación con el suelo, y el precio de la vivienda... fue una verdadera masacre."

Esto no quita que la movilidad de la población juvenil haya podido tener que ver, además, con ciertos cambios en las pautas de consumo de la misma o con la imagen que traslada el barrio en relación con otras partes de la ciudad, o del exterior. En este sentido habría que destacar los comentarios que me trasladó, en una conversación informal, un profesor de idiomas del Poblenou: "Todos mis alumnos quieren marcharse del barrio cuando sean mayores. Lo ven aburrido, lleno de viejos y lejos de donde pasan las cosas [...]". ${ }^{20}$

A esto hay que añadir el comentario de David, 40 años y residente desde siempre en el barrio, cuando señalaba: "Yo cuando joven también me fui del barrio. No sólo por el precio de la vivienda, que también, sino por el ocio. El ocio aquí está muy limitado para la gente

19 Los Casals de Barri son equipamientos municipales que promueven la vida social y asociativa de la ciudad. Mayoritariamente están gestionadas por asociaciones del propio barrio. Ver Ajuntament de Barcelona, 2013. Entrevista Manel (EM) Poblenou, 24/10/2013.

20 Conversación Informal Enrico, (CIE), Poblenou, 23/05/2014 
joven. No hay sitios donde moverte, donde pasar el tiempo"21.

Ahora bien, si ha habido una pérdida del grupo poblacional más joven pero, a la vez, la población total del Distrito se ha visto aumentada, ¿de dónde provendrían esos nuevos residentes? Las estadísticas nos muestran que el incremento de la población se ha debido principalmente al grupo etiquetado como "Extranjeros", el cual ha pasado de representar solo un $2 \%$ en 1991 a un 20,1\% en 2012. Este conjunto supondría el más numeroso tras el de los catalanes, que pasa de significar un 64\% en el año de inicio de la serie, a un $59 \% 21$ años después, aunque en términos absolutos ha permanecido prácticamente estable. Le siguen en importancia los andaluces y castellano-manchegos.

De otra de las entrevistas llevadas a cabo, en esta ocasión a Clara, una vecina ${ }^{22}$ que lleva toda su vida viviendo en el barrio, podríamos sacar algún dato de interés sobre el porqué del interés despertado por el Poblenou para estos nuevos vecinos:

"El barrio ha cambiado, quizás, bueno, ha cambiado pero sigue manteniendo esa parte que nos gusta, que es como un pueblo, que conoces a la gente y tal. Ha cambiando mucho más en lo físico. Ha venido muchísimo turismo, lo que ha supuesto todo un impacto para nosotros empezar a tener tanta gente nueva aquí que no es del barrio, que utiliza los bares de otra manera... Todo esto lo ves distinto, la gente nueva que ha venido es joven, tienen hijos, y viene a este barrio porque les gusta. Han sacado muchas empresas de aquí, ha cambiado la fisionomía y, por tanto, también las relaciones. En el futuro tenemos que hacer algo para que se mantenga. Si lo dejamos en manos de urbanistas, nos quedaríamos como un reducto".

Estos nuevos residentes, jóvenes y con hijos, posiblemente de origen foráneo como nos señala la estadística, serían los que se han ocupado de mantener e incrementar la población del barrio pese, tal y como vimos antes, a la pérdida de miembros de aquellos grupos de edad más jóvenes. Destacar también que la propia población del barrio es consciente de los cambios que han ocasionado las reformas en la estructura urbana, social y productiva del Poblenou. Esto se reflejaría en las pautas y formas de consumo, esas maneras "distintas de utilizar los bares", o de sentir el propio barrio, "aburrido, lleno de viejos".

En relación a esto, es posible ver un efecto, y a la vez una señal muy clara de la profunda transformación que se ha producido en el barrio, en la apropiación y mercantilización, por parte del capital turístico e inmobiliario, de sus símbolos y de su historia, contribuyendo, como externalidad, a elevar su valor de cambio. Como ejemplo, citar la aparición reciente, a lo largo del año 2014, de una serie de folletos y mapas bajo el epígrafe The Other Poble$n o u^{23}$. El objetivo de estos materiales es mostrar ciertos atractivos del barrio que no aparecen en publicaciones tradicionales. Se trataría de otro Poblenou que está por descubrir y al que, como dicen sus promotores, solo se puede acceder mediante "the smartest guide to the catalan Manchester" 24 . De esta forma, el barrio se revelaría como un destino maduro donde esta empresa ha visto la oportunidad de reivindicar (y crear) el off-Poblenou, lo que no deja de ser más que otra vuelta de tuerca a su conversión en un referente para el consumo.

Sin embargo, la cosa no queda aquí. La misma guía expone algunas referencias a ciertos espacios olvidados del barrio con el objetivo de añadirlos a su menú de ofertas. Entre estos espacios, y bajo el epígrafe de "Records del Segle XIX", aparece la Torre de las Aigües del

21 ED (Poblenou), 10/01/2014

22 EC (Poblenou), 21/10/2013.

23 Editados por la empresa Sömi Graphic Studio. Esta empresa informa en su página web que se trata de "un equipo de diseño gráfico flexible y comprometido en cada proyecto". www.somistudio.com

24 www.theotherpoblenou.com 


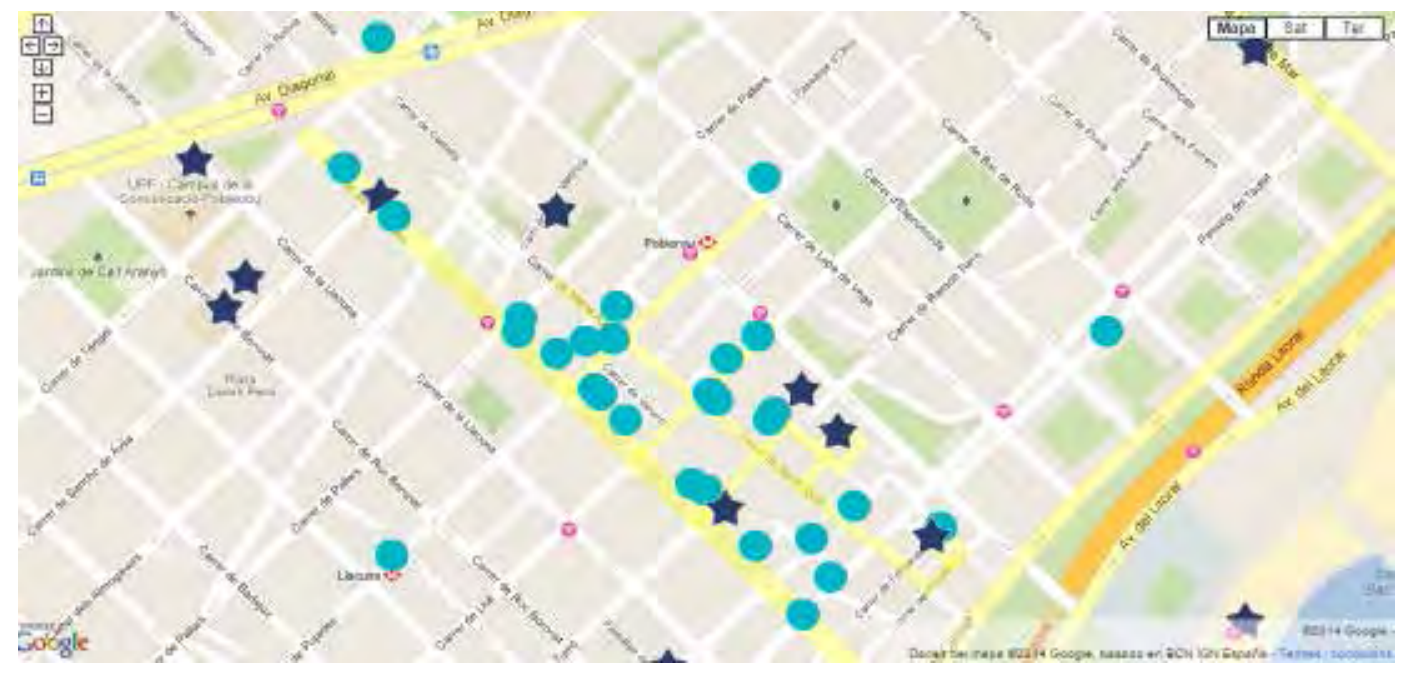

Mapa $\mathbf{n}^{\circ 2}$. Establecimientos que aparecen recogidos en el mapa The Other Poblenou Fuente: www.theotherpoblenou.com

Besòs, antiguamente propiedad de la empresa Material y Contrucciones S.A. (MACOSA). Se trata del último vestigio de un espacio fabril que contaba con más de 30 hectáreas y que se convirtió, a inicios del siglo XXI y mediante una oportunda recalificación urbanística, en parte del barrio de Diagonal $\mathrm{Mar}^{25}$, área de la que ya hemos hecho oportunda referencia en párrafos anteriores.

Otro de los referentes que aparecen en dicha guía es la Flor de Maig, antigua cooperativa de consumo, fundada por 16 obreros de la industria de las botas de vino, y que llegó a convertirse en un referente para el cooperativismo catalán de principios del siglo XX (Marín, 2006). Sobre este edificio la guía comenta que se trata de un espacio "autogestionat pels veïns de forma assembleària amb l'objectiu de portar a terme una recuperació de la memòria històrica del Poblenou, així com crear un espai de trobada pels col-lectius i entitats del barri i de la ciutat" (The Other Poblenou). La Flor de Maig es, hoy en día, un Ateneu popular del barrio, un espacio gestionado por los vecinos que decidieron okuparlo en octubre de 2012 y destinarlo a finalidades de carácter social, político y cultural con un claro mensaje contra el modelo de ciudad que se estaba imponiendo (Ateneu la Flor de Maig, 2012).

Estos ejemplos van en la línea que señalara Manuel Delgado, cuando, para explicar la atracción que determinadas clases sentían por este tipo de barrio, señalaba la necesidad de "reapropiación (...) por parte de clases medias y altas ansiosas de un baño de venerabilidad histórica, debidamente sazonada con elementos de ese nuevo sabor local que da el multiculturalismo e incluso de un moderado toque canalla" (2006).

El capital turístico e inmobiliario aparecería así como una gigante máquina devoradora de las memorias y los recuerdos del barrio al servicio de la plusvalía. Como señalaba Cla$\mathrm{ra}^{26}$, vecina, en diágolo informal: " $S$ 'ho apropien tot, converteixen la lluita en una fira $i$ ara es creuen els més guais per tenir al barri espais autogestionats"

Áhora bien, ¿quiénes son esas clases medias?, ¿qué características las hacen diferentes?, ¿dónde se hallan ubicados? Realizaré una primera aproximación a las mismas a través del estudio de los datos relativos a la renta familiar. A mayor renta se entenderá trabajadores

25 Diagonal Mar no solo es un amplio sector de viviendas de gama media-alta, sino que también cuenta con un parque diseñado por Enric Miralles, un centro comercial y espacio destinado a oficinas y hoteles.

26 CIC (Poblenou), 21/10/2013. 
mejor pagados, o pequeños empresarios y, por tanto, una posición mayor dentro de la estructura social local. Posteriormente intentaré realizar un análisis espacial de los mismos.

El examen del periodo 2008-2012, para los distintos barrios que conforman el Distrito de Sant Martí, nos muestra una situación dispar en la distribución de las rentas. Mientras que en los barrios de la Vila Olimpíca y Diagonal i el Front Marítim del Poblenou, ésta supera la media de la ciudad de Barcelona (considerada como 100), el resto de barrios permanece por debajo de la misma. Concretamente, el Poblenou pasa de tener una renta media del 93,6, para el año 2008, al 89,8 para el último año considerado. Mientras, para los barrios antes señalados, esta pasa del 133,3 y 104,4, al 146,6 y 127,7 respetivamente, lo que no deja de ser una señal del aumento de las disparidades y las diferencias dentro del mismo Distrito ${ }^{27}$ y de cierta concentración de aquellos grupos con mayor poder adquisitivo en estos barrios de nueva creación.

Los cambios han sido también evidentes para los vecinas y vecinos del Poblenou. En otra de las entrevistas llevadas a cabo, en esta ocasión a Josep Lluis ${ }^{28}$, activista con más de 20 años de lucha vecinal, éste nos señalaba como:

"Los vecinos del barrio eramos conscientes de que con las transformaciones urbanísticas y productivas que se estaban produciendo, todo el paso de una industria clásica, desde los talleres a las nuevas tecnológicas, iba a cambiar la estructura tradicional del barrio, porque iba a venir otro tipo de gente. Pero nosotros haciamos una crítica al modelo de ciudad, sobre todo vinculado al 22@. Y se ha demostrado, por el tema de ciudad, de industria que se ha promovido y se ha destruido. Era una visión, desde el barrio, muy crítica y muy a la expectativa a la transformaciòn del barrio".

Ahondemos en esta caracterización a través de los precios de la vivienda. Para el periodo comprendido para los años 2009 a 2012, el precio medio del $\mathrm{m}^{2}$ de la vivienda en Barcelona ha sido de 3.727 euros, mientras que, dentro del Distrito de Sant Martí, éste ha sido de 5.007,75 euros para Diagonal Mar i Front Maritim, 3.987 euros para la Vila Olímpica ${ }^{29}$, 3.792,75 euros para el Poblenou, 3.448,75 euros para Provençals del Poblenou, 3.181,50 euros para Camp de l'Arpa del Clot, 3.144,25 euros para el Clot, 3.039,50 euros para el Parc i la Llacuna, y 2.515 euros para el Besòs i el Maresme. Los precios del suelo superan a la media de Barcelona en los barrios de Diagonal i Front Matirim, Vila Olímpica y el Poblenou. Si al alto nivel de precios de la vivienda, superior en el Poblenou en un $2 \%$ a la media de la ciudad, le unimos la caída en el nivel de renta familiar, de un 3,8\% en los años 2008 a 2012, podríamos estar ante uno de los factores que han favorecido la pérdida de población juvenil del barrio ${ }^{30}$.

De nuevo nos acercamos al trabajo de campo para recoger la impresión que dos jóvenes del barrio, Biel y Roser ${ }^{31}$, tienen con respecto a los cambios que ha vivido el Poblenou. Aportan, por otro lado, una perspectiva interesante ya que unen en sus consideraciones aspectos relativos al desplazamiento de los más jóvenes junto a cuestiones relacionadas con los nuevos vecinos del barrio:

27 La propia prensa, durante los últimos años, se ha encargado de poner de manifiesto esta creciente disparidad. Ver La Voz de Barcelona, 2013.

28 Entrevista JL (Poblenou), 09/05/2014

$29 \mathrm{El}$ único dato ofrecido por el Ayuntamiento para este barrio se corresponde con el año 2013.

30 En un contexto de paro juvenil alto (la EPA del año 2012 mostraba un paro para menores de 25 años del $54,8 \%$ para la ciudad de Barcelona), y con precios mucho más accesibles para la vivienda en otros barrios de la ciudad, es lógico que los jóvenes busque vivienda fuera del Poblenou.

31 Entrevista BiR (Poblenou), 08/02/2014 
“Es tracta d'un barri popular però que en els darrers temps està canviant fins una cosa que no és el barri social i obrer. Els plans urbanistics van crear diferents barris, a aquests nous espais, han entrat gent que no té una identitat del Poblenou. El fet d'estar aprop de la costa i l'apuesta de l'Ajuntament pel turisme, les coses de qualitat i no se qué, està fent que els joves de tota la vida del Poblenou hagin tingut que marxar i entrin parelles o gent ambn més diners o més estabilitat econòmica. El canvi més important és la Vila Olimpica, però la part de Diagonal Mar i el Fòrum ès impresionant".

Completaremos la descripción realizada mediante el análisis del nivel de estudios, los mayores niveles de formación que revelan los diferentes barrios del Distrito para el año 2012. Los barrios de la Vila Olímpica del Poblenou y Diagonal i Front Maritim muestran los mayores porcentajes de población con "Estudios Universitarios o de Ciclos Formativos de Grado Superior (CFGS)", con un 51,4\% y un 38\% respectivamente. A gran distancia le sigue el Poblenou con un 26,7\% y, en último lugar, se sitúa el Besòs i el Maresme con un $8,3 \%$.

El retrato que nos muestran los datos relativos a nivel de renta familiar, precio de las viviendas y nivel de estudios es claro y sitúa en aquellos nuevos barrios que surgieron en antiguos emplazamientos fabriles (Diagonal Mar i From Maritim del Poblenou y la Vila Olímpica) a aquellos grupos sociales, las clases medias y medias-altas, con mayores niveles de ingresos, viviendas de mayores precios y mayores niveles de estudio.

Esto quedaría reflejado en los comentarios realizados por Carla, profesora de instituto jubilada que vino a vivir al From Maritim del Poblenou en 1998:

"Es un barrio muy agradable, donde se vive de una forma muy tranquila. Tiene ventajas, está al lado del mar y tiene muchos espacios para pasear. Es un barrio que se ha revalorizado. Antiguamente era luchador, obrero [...] pero ahora ha venido toda una serie de gente, sobre todo a partir de su renovación, y el tipo de crecimiento que ha tenido ha estado basado en un tipo de gente joven, profesional, que le ha dado un carácter determinado. Se trata de un barrio amable para vivir" 32

En definitiva, un barrio amable, o barrios amables, que se estarían constituyendo en base a las prácticas socioespaciales que protagonizarían sus residentes y que podría llegar a ocasionar conflictos por las contradicciones existentes entre su valor de uso y de cambio. Como nos señalara Loles, joven residente en el barrio y casada con un vecino de los de toda la vida:

"El tipo de gente que se queda en un barrio determina el carácter de dicho barrio. En el Poblenou podrá haber un tipo de gente más politizado, universitario e intelectual, mientras que en otros barrios es más obrera [...]. Ahora mismo el barrio asiste a una turistificación. El Ayuntamiento no se está gastando ni una pela en el barrio, la inversión que se está llevando a cabo en el Poblenou es eminentemente privada. Por ejemplo, desde las asociaciones de comerciantes, etc., y esto puede llegar a ocasionar conflictos entre los distintos intereses implicados". 33

Conflictos que ya están aquí, como lo demuestran los hechos ocurridos el 10 de abril de

32 Entrevista ECA (Poblenou), 02/05/2014

33 Entrevista EL (Poblenou), 29/05/2014 
2013 cuando el Distrito de Sant Martí dió ordenes de comenzar unas obras de modificación de la Rambla del Poblenou. La idea original, nacida en las instancias municipales con el apoyo de los comerciantes del barrio, era aumentar el espacio destinado en la Rambla a terrazas de bares y restaurantes, ampliando para ello las rotondas y reduciendo el tráfico, no solo de coches, sino también de viandantes. El día señalado, unos veinticinco vecinos y vecinas salieron a la calle con el objetivo declarado de detener el comienzo de las obras. Tras una conversación breve de estos con los trabajadores de la contrata, y una posterior llamada a la sede del Distrito, éste decidió detener las obras y que la empresa se marchase. Los vecinos y vecinas, conscientes de que después de este primer paso habría que hacer alguna cosa, convocaron ese mismo día a una reunión abierta a todos aquellos interesados en nuevas acciones, no solo reivindicativas, sino también propositivas. Dio comienzo así un proceso participativo que, tras 8 meses de trabajo, fue capaz de elaborar una propuesta para la Rambla alternativa a la del Ayuntamiento. Algo que éste, tras diversos tiras y aflojas, no tuvo más remedio que aceptar (Mansilla, 2013).

\section{A modo de Conclusión}

Cincuenta años después del nacimiento del concepto, y tras largos y encendidos debates en torno a su naturaleza y definición exactas, si hay una cosa que parece estar clara en torno a la gentrificación es que no se trata de una fenónemo aislado, sino un elemento inserto en una dinámica más amplia y general que se viene produciendo en las ciudades bajo la égida del neoliberalismo (Harvey, 2007).

En un mundo globalizado, y Barcelona es un claro ejemplo de ello, las ciudades compiten entre sí por la atraccion de nuevas inversiones y capitales (Sassen, 1999). De esta forma, es fácil hoy en día encontrar adjetivos que califican a las ciudades como inteligentes, resilientes, sostenibles, etc., epítetos sobre los cuales se encuentra el deseo último de hacerlas más atractivas, dotándolas de contenido y significado en un intento de convertirlas en mercancia. En esta dinámica juega un papel fundamental el urbanismo. Como nos recordara el sociólogo francés Henri Lefebvre (1972), bajo una apariencia tecnológica, humanista y positiva, el urbanismo esconde lo que realmente es, un instrumento destinado al control del espacio bajo el capitalismo. La gentrificación aparecería así como un eslabón más en la cadena de extracción de plusvalías de las ciudades por medio de la explotación de las rentas monopolísticas que ésta genera.

En el caso de estudio, el barrio del Poblenou, en Barcelona, la gentrificación no se ha producido tanto por la reapropiación, por parte de aquellas clases con mayor poder adquisitivo, de antiguos edificios de viviendas rehabilitados, sino por la ocupación por parte de esas clases de nuevas construcciones realizadas sobre los solares que una vez ocuparon viejas industrias locales. En este sentido, el proceso es diferente al ocurrido en otros barrios de la misma ciudad, como Gracia o el Born (Mansilla, 2014b). La recuperación de antiguos terrenos industriales, o brownfield redevelopment, con el objetivo de situar sobre ellos promociones inmobiliarias, así como empresas que contribuyan a reorientar la actividad productiva local siguiendo un diseño preestablecido desde las instancias municipales, ha creado diferentes unidades territoriales y administrativas, nuevos barrios, donde hace poco más de una década existía un único, más o menos social y económicamente homogéneo, Poblenou.

De este modo, la población del Distrito, y del barrio, no solo no ha disminuido, sino que se ha visto incrementada. Este aumento se debe, fundamentalmente, a aquellos vecinos y vecinas que se han ido incorporando a los distintos barrios en los que se ha subdividido el antiguo Poblenou. Por otro lado, estos nuevos residentes son diferentes a los anteriores. Jóvenes con familia, profesionales, extranjeros, universitarios, intelectuales, etc., son algunos de los adjetivos y expresiones con los que podríamos definir estos nuevos grupos sociales. Si quisiéramos encontrar un denominador común para dichos grupos este vendría dado, 
además de por sus mayores ingresos y formación, tal y como nos muestra la estadística, por nuevas formas de consumir y de vivir. Además, los nuevos residentes se encuentran espacialmente localizados. El Distrito de Sant Martí surge como un mosaico diverso, con claras diferencias entre zonas separadas, la gran mayoría de las veces por una única calle, y que una vez conformaron una única unidad. Aquellas áreas de nueva construcción, como la Vila Olimpica, Diagonal Mar i el Front Maritim, aparecerían como bloques uniformes habitados por gentes de clase media y media-alta, diferentes socialmente a los habitantes del resto de barrios del Distrito.

El barrio ha visto desaparecer a sus jóvenes. Estos, o no han podido acceder a una vivienda en el mismo debido a los niveles que ha alcanzado el precio del suelo, así como a la precariedad generalizada, o no lo encontrarian atractivo. El Poblenou se aparecería así como un espacio para el consumo de clases medias jóvenes y profesionales que aportan nuevas formas de relacionarse, de vivir el barrio y la ciudad.

Por otro lado, destacar la mercantilización de los símbolos del barrio, de su patrimonio intangible y sus restos industriales, algo que incide en la conversión del mismo en un espacio de consumo para turistas y residentes y que podría llevar, como nos muestran el ejemplo de la detención de las obras de remodelación de la Rambla del Pobneou, a la generación de numerosos conflictos por el espacio.

Por último, señalar la posibilidad que ofrece, a la hora de establecer alianzas en estos conflictos, el considerar a las clases sociales, no tanto en función de determinadas categorías escalares o descriptivas, sino en cuanto a su posición, en el sentido amplio de la palabra, en el proceso de creación de valor de todo proceso de gentrificación. Así, las formas en las que la economía neoliberal extrae las rentas de la ciudad sitúa a grupos, otrora heterogéneos, ante la opotunidad de organizarse y ofrecer respuestas conjuntas ante estos desafíos.

En definitiva, nos encontramos ante un proceso dialectico que se encuentra determinado por la acción de dos elementos distintos pero íntimamente interrelacionados: las transformaciones urbanas y los grupos sociales que actúan según las mismas.

\section{Bibliografía}

ALIBÉS, Josep María et al

1975 La Barcelona de Porcioles. Barcelona: Ed. Laia.

ARBONA, Javier.

2014 "Happy Fifty Years, Gentrification! Does Gentrification Gentrify without Gentrifiers?", en The Polis Blog. http://www.thepolisblog.org/2014/02/happy-fifty-years-gentrification. html (15 septiembre 2014).

ARROYO, Francesc

1999 "Barcelona construyó la Villa Olímpica sobre un área industrial degradada" en El País http://elpais.com/diario/1999/11/19/andalucia/942967331_850215.html (15 septiembre 2014).

ATENEU LA FLOR DE MAIG

2012 "Manifest per l'okupació", en Ateneu la Flor de Maig. http://ateneuflordemaig.wordpress. com/manifest-2/ (15 septiembre 2014).

AYUNTAMIENTO DE BARCELONA

2000 Modificació del PGM per la renovació de les zones industrials del Poblenou - Districte d'Activitats 22@BCN, en bnc.cat. http://www3.amb.cat/normaurb2004/Docs/Normes_ mod/NUM-Barcelona-5.pdf (15 septiembre 2014).

2006 Departament d'Estadistica, en bcn.cat http://www.ben.cat/estadistica/castella/index.htm (15 septiembre 2014).

2013 Centros Cívicos y Casales de Barrio, en bcn.cat. http://w110.bcn.cat/portal/site/Joves/menuitem.fcc7c99428df85297ca47ca4a2ef8a0c/?vgnextoid=74eca29f5a6a8210VgnVCM1 0000074fea8c0RCRD\&vgnextchannel=74eca29f5a6a8210 VgnVCM10000074fea8c0RC RD\&lang=es_ES (15 septiembre 2014). 
CAPEL, Horacio

2005 El modelo Barcelona: un examen crítico. Barcelona: Ediciones del Serbal.

2007 "El debate sobre la construcción de la ciudad y el llamado Modelo Barcelona"”, en Scripta Nova. Revista Electrónica de Geografía y Ciencias sociales, Vol. XI, 233 http://www. ub.es/geocrit/sn/sn-233.htm (15 septiembre 2014).

CAULFIELD, Jon

1989 "Gentrification and desire", en Canadian Review of Sociology and Anthropology, 26(4): 617-632

CHARNOCK, Greig et al

2014 "City of rents: The limits to the Barcelona model of urban competitiveness", en International Journal of Urban and Regional Research, Vol. 38.1: 198-217.

DAVIDSON, Justin

2014 Is gentrification all bad?, en New York Magazine. http://nymag.com/news/features/gentrification-2014-2/ (15 septiembre 2014).

DELGADO, Manuel

2005 Elogi del vianant. Del Model Barcelona a la Barcelona real. Barcelona: Edicions de 1984.

2006 "El forat de la vergonya", en El País. http://elpais.com/diario/2006/10/10/catalunya/1160442449_850215.html (15 septiembre 2014).

2007 La ciudad mentirosa. Fraude y miseria del Modelo Barcelona. Barcelona: Libros de la Catarata.

GARCÍA HERRERA, Luz Marina

2001 "Elitización: Propuesta en español para el término gentrificación", en Biblio 3W Revista bibliográfica de Geografía y Ciencias Sociales, Vol. VI, 332. http://www.ub.edu/

GLASS, Ruth geocrit/b3w-332.htm (15 septiembre 2014).

1964 London Aspects of changes. London: Ed. MacGibbon and Klee.

FERNÁNDEZ, Miquel

2012 Matar al Chino. Entre la revolución urbanística y el asedio urbano en el barrio del Raval de Barcelona. Barcelona: Universitat de Barcelona. http://diposit.ub.edu/dspace/handle/2445/35237 (15 septiembre 2014).

FRANQUESA, Jaume

2007 "Vaciar y llenar, o la lógica espacial de la neoliberalización”, en REIS. Revista Española de Investigaciones Sociológicas, 118: 123 - 150.

GARCIA-RAMÓN, Maria-Dolors, ALBET, Albel

2000 "Pre-olympic and post-olympic Barcelona, a 'model' for urban regeneration today?", en Environment and Planning A, 2000, vol. 32: 1331-1334.

GAUDEMAR, de Jean Paul

1991 El orden y la producción. Nacimiento y formas de disciplina en la fábrica. Madrid: Ed. Trotta.

GINER, Salvador

1981 Sociología. Barcelona: Ed. Península.

HARVEY, David

1977 Urbanismo y desigualdad social. Madrid: Ed. Siglo XXI.

1989 "From Managerialism to Entrepreneurialism: The Transformation in Urban Governance in Late Capitalism", en Geografiska Annaler, Series B, Human Geography, Vol. 71, 1: 3-17.

2001 Espacios del capital. Hacia una geografía crítica. Madrid: Ed. Akal.

2007 Breve historia del neoliberalismo. Madrid: Ed. Akal.

2013 Ciudades rebeldes. Del derecho a la ciudad a la revolución urbana. Madrid: Ed. Akal.

2014 Seventeen contradictions and the end of the capitalism. London: Profile Books.

HUERTAS CLAVERIA, Josep Maria, MARTÍ, Jose y FABRE, Jaume

1976 Tots els barris de Barcelona. Barcelona: Ed. 62.

Instituto Nacional de Estadística

2014 Encuesta primer trimestre, en INE. http://www.ine.es/daco/daco42/daco4211/epa0114. pdf (15 septiembre 2014). 


\section{LA VOZ DE BARCELONA}

2013 "Aumentan las desigualdades entre los barrios ricos y los pobres de Barcelona", en $L a$ Voz de Barcelona. http://www.vozbcn.com/2013/01/06/136981/aumentan-desigualdadesbarrios-barcelona/ (15 septiembre 2014).

\section{LEEDS, Anthony}

1994 Cities, classes and social order. New York: Cornell Univeresity

LEFEBVRE, Henri

1972 La revolución urbana. Madrid: Ed. Alianza.

LÓPEZ SÁNCHEZ, Pere

1990 "Normas e ilegalismos. El control social y los usos del territorio en la metrópoli", en Horacio Capel (coord.), Los espacios acotados. Geografía y dominación social: 167-197. Barcelona: Promociones y Publicaciones Universitarias

LLOYD, Richard

2002 "Neo-bohemia: Art and neighborhood redevelopment in Chicago", en Journal of Urban Affairs, Vol. 24: 517-537.

MANSILLA LÓPEZ, José Antonio

2013 "Contra la manca de participació, acció veïnal. El cas de la Rambla del Poblenou” en $L a$ Directa, n'334: 10.

2014a "La Flor de Maig somos nosotros. Geografía urbana de la memoria en el Poblenou, Barcelona", en Actas el XIII Coloquio Internacional de Geocrítica, El control del espacio y los espacios de control, 5/10 de mayo de 2014. Universitat de Barcelona. http://www. ub.edu/geocrit/coloquio2014/Jose\%20Antonio\%20Mansilla\%20Lopez.pdf (15 septiembre 2014).

2014b "Hasta conseguirlo. Traición a la tradición en el Born Centre Cultural", en Eldiario.es Catalunya Plural. http://www.eldiario.es/catalunya/opinions/conseguirlo-Traicion-BornCentre-Cultural_6_305929439.html (15 octubre 2014).

2014c "Multifuncionalidad y externalidades en contextos urbanos. Ideas para una política antigentrificación", en Working Paper Series - Contested Cities, WPCC-14011, Serie (I), Gentrificación, resistencias y desplazamiento en España. http://contested-cities.net/working-papers/2014/multifuncionalidad-y-externalidades-en-contextos-urbanos-ideas-parauna-politica-antigentrificacion/ (15 septiembre 2014).

MARÍN, Doloeres

2006 "De 1890 a 1939, La cooperativa La Flor de Maig”, en Flor de Maig: del cooperativisme al servei als municipis: 19-70. Barcelona: Diputació de Barcelona

MARRERO, ISAAC

2003 “Del Manchester catalán al Soho Barcelonés? La renovación del barrio del Poblenou en Barcelona y la cuestión de la vivienda”, en Scripta Nova, Revista Electrónica de Geografía y Ciencias Sociales, Vol. VII, 146(137). http://www.ub.edu/geocrit/sn/sn-146(137).

MARX, Karl htm (15 septiembre 2014).

2003 El capital: crítica de la economía política. Barcelona: RBA coleccionables.

MORELL, Marc

2013 "De l'espai no t'en refies mai”: el treball urbà en la formació/lluita de clase", en ICA, Quaderns-e, 18 (2): 53-67. http://www.antropologia.cat/files/Quaderns-e\%2018(2)_ article4(Dossier1).pdf (15 septiembre 2014).

NEVIUS, C.W.

2014 "Gentrification no longer a dirty Word" en San Francisco Gate (SFGate.) http://www.sfgate.com/bayarea/nevius/article/Gentrification-no-longer-a-dirty-word-4302093.php (15 septiembre 2014).

ROSE, Damaris

1984 "Rethinking gentrification: beyond the uneven development of urban marxist theory", en Environment and Planning: Society and Space, vol. 1: 47-74.

SASSEN, Saskia

1999 Ciudades globales. Nueva York, Londres, Tokio. Buenos Aires: Ed. Eudeba. 
SENNET, Richard

1997 Carne y piedra. El cuerpo y la ciudad en la civilización occidental. Madrid: Ed. Alianza. SHAW, Kate.

2008 "Gentrification: what it is, why it is, and what can be done about it", en Geography Compass 2: 1-32.

SLATER, Tom

2011 "Gentrification of the city", en The New Blackwell Companion to the City: 571-585. Lon-

SMITH, Neil dres: Ed Blackwell Publishing Ltd.

2002 "New globalism, new urbanism: gentrification as global urban strategy", en Antipode, 34

SMITH, Neil (3): 427-450.

2012 La nueva frontera urbana. Ciudad revanchista y gentrificación. Madrid: Traficantes de TELLÓ, Rosa Sueños.

1993 "Barcelona post-olímpica: de ciudad industrial a escenario de consumo", en Estudios Geográficos, Vol. 54, 212: 507-520.

TREANOR, Paul

2004 "Brownfield gentrification in Amsterdam", en web.inter.nl http://web.inter.nl.net/users/ Paul.Treanor/gentrification.html (15 septiembre 2014).

UTRERA, Joaquima

2001 "El Poblenou quiere servicios antes del aluvión", en El País. http://elpais.com/diario/2001/08/18/catalunya/998096844_850215.html (15 septiembre 2014). 